This item was submitted to Loughborough's Research Repository by the author.

Items in Figshare are protected by copyright, with all rights reserved, unless otherwise indicated.

\title{
Non-aqueous nanofiltration: solute rejection in low-polarity binary systems
}

PLEASE CITE THE PUBLISHED VERSION

PUBLISHER

(C) Elsevier

VERSION

AM (Accepted Manuscript)

LICENCE

CC BY-NC-ND 4.0

REPOSITORY RECORD

Tarleton, E.S., J.P. Robinson, C.R. Millington, and Arian Nijmeijer. 2009. "Non-aqueous Nanofiltration: Solute Rejection in Low-polarity Binary Systems”. figshare. https://hdl.handle.net/2134/4788. 
This item was submitted to Loughborough's Institutional Repository (https://dspace.lboro.ac.uk/) by the author and is made available under the following Creative Commons Licence conditions.

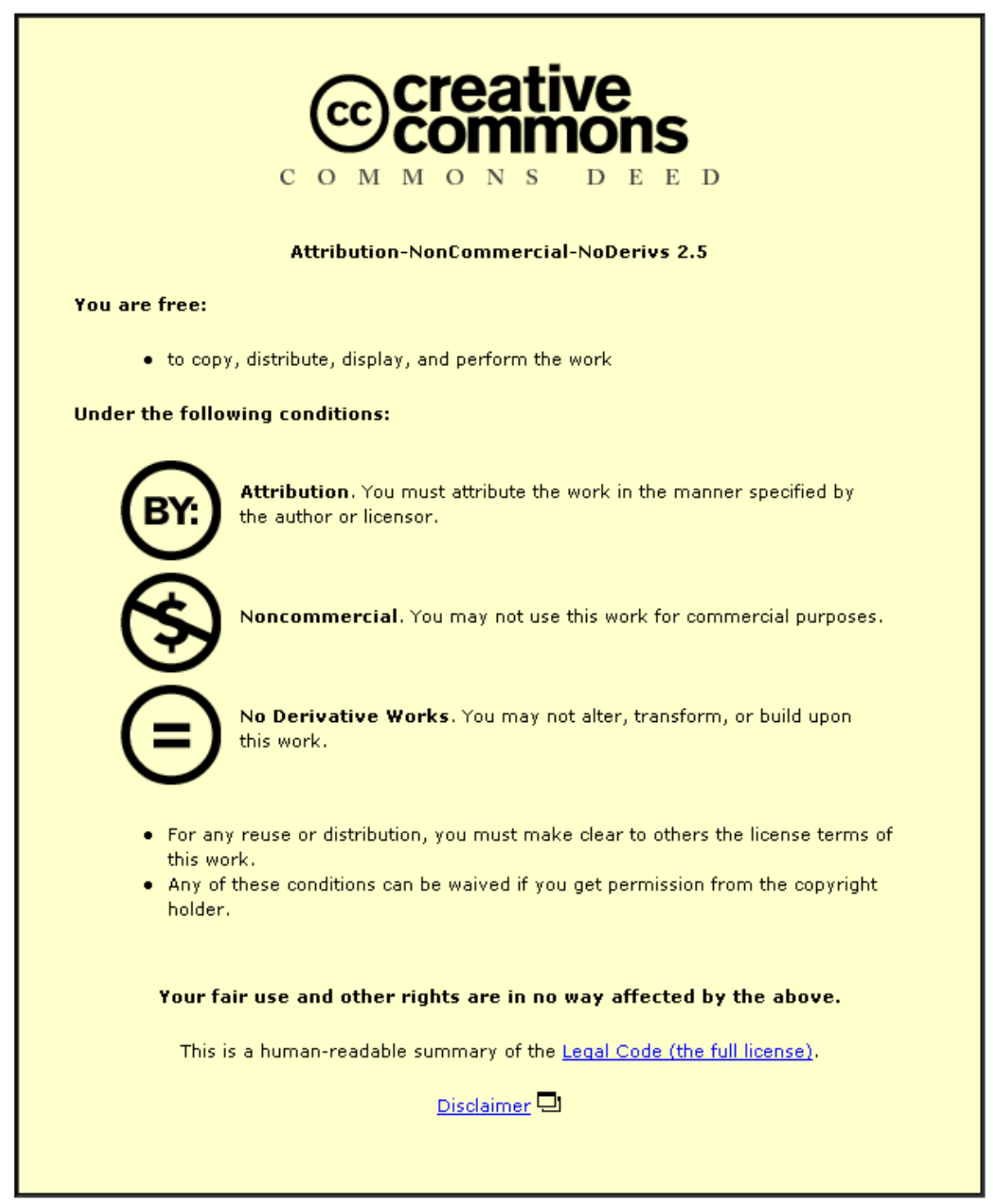

For the full text of this licence, please go to: http://creativecommons.org/licenses/by-nc-nd/2.5/ 


\title{
NON-AQUEOUS NANOFILTRATION: SOLUTE REJECTION IN LOW-POLARITY BINARY SYSTEMS
}

\author{
E.S. Tarleton ${ }^{1}$ (e.s.tarleton@lboro.ac.uk), J.P. Robinson ${ }^{1}$, C.R. Millington ${ }^{2}$ and A. Nijmeijer ${ }^{3}$ \\ ${ }^{1}$ Advanced Separation Technologies Group, Department of Chemical Engineering, Loughborough \\ University, Loughborough, Leicestershire LE11 3TU, UK. \\ ${ }^{2}$ Shell Global Solutions, Cheshire Innovation Park, P.O. Box 1, Chester CH1 3SH, UK. \\ ${ }^{3}$ Shell Global Solutions International BV, P.O. Box 38000, 1030 BN Amsterdam, The Netherlands.
}

\begin{abstract}
The separation characteristics of a dense polydimethylsiloxane (PDMS) membrane were studied using alkyl and aromatic solvents and low-polarity, sulphur bearing, organometallic and polynuclear aromatic (PNA) solute compounds. Rejection was found to be dependent on transmembrane pressure, crossflow rate (hydrodynamic conditions), solute size and the degree of swelling induced by the solvent. Rejection increased progressively with pressure whilst a threshold condition was observed above which further increases in crossflow had a negligible influence on rejection. Measurements over the molecular weight range $84-612 \mathrm{~g} / \mathrm{mol}$ showed the membrane to have a molecular weight cut-off in the region $350-400 \mathrm{~g} / \mathrm{mol}$ to all but one of the tested PNA compounds (i.e. rubrene). An additional correlation using molecular dimensions instead of molecular weight showed the cut-off size of the membrane to be in the region of 1-2 $\mathrm{nm}$, with all data falling on a well defined rejection/size curve.
\end{abstract}

Solvent type influenced membrane swelling to an extent dependent on the relative magnitude of the solubility parameters for the solvent and PDMS; similar values led to more swelling, higher fluxes and lower rejections. Results support the concept of viscous solvent flow whilst solute transport could be either predominantly viscous or a combination of viscous and diffusive. With larger molecules a size exclusion mechanism was dominant. A new model is proposed that takes account of solute transport by a combination of viscous and diffusive mechanisms and this is shown to well represent the experimental data.

\section{KEYWORDS}

Nanofiltration; Membrane; PDMS; Non-aqueous; Solute rejection; Polymer swelling; Filtration; Organometallic; PNA

\section{INTRODUCTION}

In recent years the feasibility of using polymeric nanofiltration (NF) membranes for non-aqueous separations has been explored, examples include the recovery of organometallic catalysts from organic solvents ${ }^{1}$ and the de-acidification of vegetable oils ${ }^{2}$. There is, however, limited understanding of the fundamental mechanisms involved. The literature favoured concept for nonaqueous NF systems is the solution-diffusion model first proposed by Lonsdale et al. ${ }^{3}$, where permeation takes place by a substance dissolving in the membrane material and subsequently diffusing through it. The selectivity of the membrane is governed by differences in the solubility and diffusivity of the permeating species, with the sorption behaviour being generally non-ideal ${ }^{4,5}$. An alternative approach is the pore flow model, where even a dense membrane is considered to behave as if it had an appreciable free volume and pores through which viscous permeation takes place.

Currently of principal interest are NF membrane composites incorporating a relatively thin separating layer of polydimethylsiloxane (PDMS). This relatively robust polymer is stable in the 
presence of many organic solvents, although the passage of solvent(s) induces physical, but reversible, swelling. The Hildebrand solubility parameter, $\delta$, is reported to be a good indictor of the extent of swelling to be expected for a particular solvent/PDMS membrane combination ${ }^{6,7}$, solvents with a solubility parameter similar to that of PDMS (i.e. $\delta=15.5 \mathrm{MPa}^{0.5}$ ) induce more swelling. Hydrophobic membranes such as PDMS have a low value of $\delta$, and as such will be swollen by lowpolarity solvents with similarly low values of $\delta$.

Although the literature in non-aqueous NF is by no means extensive, several workers have studied and attempted to model the solute rejection behaviour of relevant non-aqueous systems.

Scarpello et al. ${ }^{1}$ studied the recovery of organometallic catalysts from solvents with a range of polarities using, amongst others, an MPF-50 (essentially PDMS) membrane. They found that catalyst rejections were close to unity and dependent on the solvent type. The rejection was reported to increase with pressure and postulated that compaction of the membrane layer resulted in a smaller effective pore size. This hypothesis was seemingly contradicted by the authors themselves as the measured solvent flux-pressure relationship remained linear over the tested pressure range. van der Bruggen et al. ${ }^{8}$ also studied solute rejection with an MPF-50 membrane, and again reported the extent of solute rejection to depend on the solvent type. Interestingly, the rejection in low-polarity solvents was found to be reduced when compared with that observed with polar solvents. Gibbins et al. ${ }^{9}$ showed solute rejection in methanol to increase with both pressure and solute molecular weight, and estimated an effective pore diameter of $\sim 1.2 \mathrm{~nm}$ for the MPF-50 membrane based on available pore models. Bhanushali et al. ${ }^{6,10}$ studied a range of solvents and solutes with both hydrophilic and hydrophobic membranes; the latter comprised PDMS composites at least superficially similar to the membranes used in the current study. Through experimental observations and the application of existing models, the authors concluded that convective transport can be significant and coupling of solvent and solute flows may take place. Other workers, for example, Zwijenburg et al. ${ }^{2}$, Raman et al. ${ }^{11}$, White ${ }^{12}$ and Stafie et al. ${ }^{13}$ have also examined hexane/oil and hexane/aromatic systems, albeit in some cases with non-PDMS, more polar, membranes.

Several of the quoted works tested solvents and solutes that are significantly more polar than those employed in the current study. In some cases solute concentrations are also significantly higher. Although representative of some potential applications, polar solvents and solutes may interact significantly, both with each other and with the separating membrane, which can make it difficult to isolate the effect of either substance and to understand the transport mechanisms. In the study reported here low-polarity solvents and low, but representative, concentrations of solute have been used to both enhance the understanding of non-aqueous NF processes and assess the potential for applying the technology in a new industrial sector.

\section{EXPERIMENTAL}

\section{Membrane}

All experiments used a polyacrylonitrile (PAN)/polydimethylsiloxane composite membrane supplied by GKSS Forschungszentrum. The PAN substrate has previously been shown to be inactive ${ }^{7}$ whilst the radiation crosslinked and selective PDMS layer exhibited a nominal thickness of $2 \mu \mathrm{m}$. A single membrane was used to obtain the reported data and no preconditioning of the membrane was required prior to an experiment (repeat experiments with nominally identical membranes yielded similar results to those shown). No appreciable deterioration in flux/rejection performance was observed over the duration of the study and individual experiments were deliberately conducted in a random order to ensure that the membrane was not conditioned by any previous solvent, solute and/or handling procedure; see also ${ }^{7,10}$. The solvent induced swelling of the composite membrane layers was completely reversible as evidenced by the sequential nature of the data presented here, periodic flux measurements with a chosen 'standard' n-heptane solvent and in-situ determinations of PDMS/PAN layer thickness to be reported at a later date. 


\section{Apparatus}

The solvent/solute systems were studied in the crossflow membrane filtration apparatus shown schematically in Figure 1.

The solvent/solute mixture was added to the 2.5 litre capacity reservoir $(A)$ from which an air-driven pump (B) delivered the fluid to the membrane module $(C)$ via a variable area flow meter $(F)$, a flow control valve (V6) and a $15 \mu \mathrm{m}$ rated pre-filter (D). The permeate could either be circulated back to the reservoir or collected separately for subsequent sample analysis. The retentate stream returned to the reservoir through a cooler $(E)$ which employed the exhaust air stream from the pump to maintain the temperature of the circulating fluid. Trans-membrane pressure and crossflow rate were controlled primarily by the back-pressure regulator (V7) and the air-regulator to the pump (V2); the flow control valve (V6) was sometimes used to make minor adjustments to process conditions. The circular, flat sheet membrane was mounted in a standard, and un-modified, Osmonics DESAL membrane module to give a wetted surface area of $75 \mathrm{~cm}^{2}$.

The maximum pressure and crossflow obtainable with this apparatus was 8 bar $(800 \mathrm{kPa})$ and 0.7 I $\mathrm{min}^{-1}$ respectively; higher solvent viscosities reduced these limits. A second apparatus was also available (on a limited basis) that facilitated filtration pressures up to 20 bar and crossflow rates of $4 \mathrm{I} \mathrm{min}^{-1}$. Although detailed specifications are not presented here, this up-rated apparatus incorporated a larger capacity pump and the same DESAL membrane module. The flow circuit was similar to that presented in Figure 1.

\section{Materials}

n-heptane, cyclohexane and xylene solvents were used in the experiments, all of which are representative of the potential application and were supplied by Sigma-Aldrich. $n$-heptane is a straight chain alkane, whilst cyclohexane and xylene (a mixture of isomers) are respectively cyclic alkane and aromatic compounds. The two solute compounds forming the main focus of the study were iron (III) acetylacetonate (an organometallic, OM) and 9,10-diphenylanthracene (a polynuclear aromatic, PNA). The chemical structures are shown in Figure 2 and these solutes were chosen for a number of reasons including:

- initial screening tests showed intermediate rejections, i.e. between 0 and 1 , which gave scope to investigate the influences of process variables on flux and rejection

- the ability to dissolve the solutes in the solvents at concentrations of up to 75 ppmw (equivalent to a mass fraction of $7.5 \times 10^{-5}$ )

- the ability to determine concentration by UV/vis spectroscopy - the absorbance of iron (III) acetylacetonate was measured at a wavelength of $450 \mathrm{~nm}$ whilst 9,10-diphenylantracene was measured at $380 \mathrm{~nm}$.

A few data were also obtained with other representative PNA, organometallic and sulphur bearing compounds (see Table 1). All solutes had initial purities in excess of $99 \%$.

\section{Procedure}

All the data reported were obtained using one litre of feed fluid in the reservoir and a $10 \%$ stage cut. Prior to an experiment the test solution was circulated at very low pressure for several minutes with no permeation to remove any trapped air in the system. With the pressure and crossflow set to the desired values, the permeate was circulated back to the reservoir for a set period in order to establish equilibrium conditions (typically 10 minutes); advice from GKSS and preliminary 'scouting' experiments had shown this period to be sufficient to establish steady state conditions and reliable values for flux and rejection measurements. The permeate was then 
diverted and collected in a separate vessel, this sample being used to determine the flux and solute concentration. All results were found to satisfy a solute mass balance to within $1 \%$.

\section{RESULTS AND DISCUSSION}

The test matrix addressed the influence of the principal operating parameters on solute rejection, namely crossflow rate, trans-membrane pressure, solute concentration, solute size and the degree of membrane swelling due to different solvent type. The latter is interlinked with the effects induced by the other operational parameters and is presented accordingly.

\section{Effects of Crossflow Rate (Hydrodynamics)}

The rejection of 9,10 diphenylanthracene was determined at a fixed pressure of $5 \mathrm{bar}(500 \mathrm{kPa})$. Three pure solvents and one solvent mixture were employed with crossflow rates ranging from 0.1-4 l/min; in the case of cyclohexane and the n-heptane/xylene mixture the crossflow was limited to $0.7 \mathrm{l} / \mathrm{min}$ due to operational difficulties with the second, up-rated apparatus. Typical results are shown in Figure 3.

In all cases the rejection increased as crossflow was raised from an initially low value, an effect also observed with the rejection of the other tested solutes. Above a threshold value of $\sim 0.5 \mathrm{l} / \mathrm{min}$, little increase in rejection was noted for $n$-heptane and xylene solvents up to the highest available crossflow rate of $4 \mathrm{l} / \mathrm{min}$. The rejection behaviour of the cyclohexane solvent was intermediate between these two pure solvents. The n-heptane/xylene mix, whose composition was arranged to give a solubility parameter of $\delta=16.8 \mathrm{MPa}^{0.5}$, yielded marginally improved levels of rejection. A potential reason for the threshold is a transition in the hydrodynamic flow regime above the membrane surface, a factor that can be characterised through Reynolds Number $(R e)$. The design of the module prevented the direct calculation of $\mathrm{Re}$ as the flow velocity and flow diameter are unknown. However, given that the module dimensions were the same in each test, and that the flow velocity is proportional to crossflow rate, $Q \rho / \mu$, a similar expression to $\mathrm{Re}$, can be used to compare rejection behaviour (see Figure 4); $Q$ is the crossflow rate, $\rho$ is solvent density and $\mu$ is solvent viscosity.

As intuitively expected, the data in Figure 4 are similar in form to those presented in Figure 3 . Whilst a threshold level of $Q \rho / \mu$ is only valid for the membrane module used in the current study, the results are significant as they show how hydrodynamics can influence separation in NF, and that an optimum crossflow rate (in terms of rejection) is just above the threshold value. Figures 3 and 4 also clearly show that solute rejection is dependent on solvent type and is a consequence of the degree of solvent induced swelling in the selective PDMS layer of the membrane. Previous work by the authors has shown how the level of solvent flux can be directly related to membrane swelling properties ${ }^{7,10}$ and the relative magnitudes of $\delta$ for the permeating solvent and membrane. The current work extends this finding whereby solvents inducing a larger amount of swelling yield lower solute rejection than poorer-swelling solvents. The solubility parameters of xylene, cyclohexane and n-heptane are $18.2,16.8$ and $15.3 \mathrm{MPa}^{0.5}$ respectively, which is consistent with the order in which the rejection data appear in Figure 4 (i.e. $\delta_{n \text {-heptane }} \approx \delta_{P D M S}$ to give the greatest swelling and lowest rejection). Further evidence that solute rejection is strongly dependent on the solvent induced swelling was obtained by testing a solvent mixture ${ }^{* *}$. Xylene and n-heptane, respectively aromatic and straight chain alkane compounds, were added together to give a mixture with a solubility parameter the same as cyclohexane, a cyclic alkane compound. Figure 4 shows that solute rejection data for cyclohexane and the n-heptane/xylene mixture overlap, not only confirming that the degree of swelling affects solute rejection, but also that solubility parameters for

\footnotetext{
${ }^{* *}$ It is noted that no separation of the solvent mixture was detectable on passage through the membrane, the resolution of the measurement being $0.2 \%$.
} 
low-polarity solvents can be used to characterise the degree of swelling of a PDMS composite membrane.

Over the ranges tested, crossflow velocity had a limited effect on permeate flux levels. Given the low levels of solute present in the feed solutions, and the lack of a surface fouling layer on the membrane, this result is not surprising. Moreover, the reversibility of the membrane in terms of performance indicators infers that any internal fouling of the PDMS layer is non-permanent. It is possible that a lower threshold level for crossflow exists, below which concentration polarisation becomes sufficient to affect both flux and rejection.

\section{Effects of Pressure}

In order to assess pressure effects over the widest range, the rejection of two solute compounds was determined in a xylene solvent with the two experimental apparatus described, both utilised the same DESAL membrane module. Rejections at pressures up to $8 \mathrm{bar}(800 \mathrm{kPa})$ were determined at a crossflow rate corresponding to $Q \rho / \mu \approx 10 \mathrm{~m}$, i.e. just above the threshold noted in Figure 4. Due to the restricted flow/pressure envelope of the up-rated apparatus, the experiments at higher pressures were performed with $Q \rho / \mu=45 \mathrm{~m}$, i.e. over four times that used at lower pressures. Figure 4 suggests that operating with such different $Q \rho / \mu$ values has a negligible influence on solute rejection and thus the results shown in Figures 5 and 6 represent the sole effects of pressure for the different solute/solvent combinations.

For both solutes the rejection improved as filtration pressure increased, with the largest gains being achieved at lower pressures. The data suggest that over the tested pressure range the rejection of 9,10 diphenylanthracene lies below that recorded for iron (III) acetylacetonate; molecular weights were 330 and 353 respectively. Although there is some scatter, the progressive blending of results from the two apparatus vindicates the comparison of data obtained above the hydrodynamic threshold. The rejection of 9,10 diphenylanthracene was further studied in a range of solvents and the results are compared in Figure 6 . In all cases the rejection improved as pressure increased, with rejection from the $n$-heptane/xylene mixture being comparable to that recorded using cyclohexane; in both these cases $\delta=16.8 \mathrm{MPa}^{0.5}$. Rejection levels with n-heptane were consistently the lowest whilst experiments with xylene displayed the highest rejections. The results confirm the indications from Figure 4 and strengthen the argument that low-polarity solute rejection is uniquely dependent on the degree of solvent induced swelling. It is noted that the data in Figure 6 are similar to those reported by Scarpello et al. ${ }^{1}$ who suggest that limiting rejection with pressure is caused by compaction of the membrane. Structural change would potentially cause flux to decline noticeably and this phenomenon was not observed in any of the data obtained by the authors. On the contrary the measured flux/pressure relationships (i.e. $J_{s} v s . \Delta P / \mu$ ) exhibited linearity over the tested pressure range with the slope being determined by the extent of solvent induced swelling. For a given solvent/solute combination, the increased rejection with pressure is apparently not solely a consequence of any change in membrane structure.

\section{Effects of Concentration}

The rejection of 9,10 diphenylanthracene was investigated with a xylene solvent at concentrations of 10-75 ppm. Although still relatively low, the upper limit of concentration is well above the normal levels found in the process fluid of interest. With reference to Figure 7, rejection is for practical purposes independent of concentration over the range studied. It is considered that solute concentrations in the ppm range are not sufficient to influence the degree of solvent induced membrane swelling, and hence the rejection remains unchanged. Intuitively, much higher solute concentrations may impact on the degree of swelling and rejection will in this case be determined, in part, by the amount of solute. A further factor that could be influenced by solute concentration is solvent flux and Figure 8 shows example data for xylene determined over the same range of concentration. Again, for practical purposes, the inference is that solvent flux is independent of solute concentration. 


\section{Effects of Solute Size}

Experiments with additional PNA, organometallic and sulphur bearing solutes were chosen to examine the influence of solute size (see Table 1). The limiting rejections of these solutes in xylene as a function of molecular weight are shown in Figure 9. With the exception of rubrene which gave a rejection lower than expected, the membrane exhibits a molecular weight cut-off in the region of $350-400 \mathrm{~g} / \mathrm{mol}$. Such behaviour is indicative of the selective PDMS layer rejecting low-polarity, minimally interacting solutes on the basis of size-exclusion. The data in Figures 3-6, and other data ${ }^{7,10}$, suggest viscous solvent transport to an extent dependent on the degree of swelling induced porosity. Increasing solute rejection with molecular weight has previously been reported by Gibbins et al. ${ }^{9}$ for an MPF-50 membrane where solute molecular weights ranged from 250 to $400 \mathrm{~g} \mathrm{~mol}^{-1}$. A size-exclusion mechanism seems unlikely for dense membranes like PDMS as solute (and solvent) transport is intuitively diffusive. Although larger molecules can be expected to have very low rates of diffusion through PDMS and thus high rejections, smaller molecules would not be expected to give zero rejections as observed in Figure 9. The latter could potentially occur through a number of scenarios, including:

- Solvent is transported via viscous flow and solute flux is diffusive. For this process to occur the solvent and solute transport rates need to be the same

- Solvent and solute fluxes both occur via a diffusive mechanism at identical rates

- Solvent and solute are transported via a viscous flow mechanism at the same rate.

In the authors' opinion, the most feasible explanation is the latter where the solvent swells the membrane to effectively induce an ill-defined porous structure in the PDMS, and the zero rejections are due to the solvent and solute moving through the membrane structure 'as one' under viscous flow with no separation occurring. Using a more limited range of solutes, very similar behaviour to that shown in Figure 9 was measured for an n-heptane solvent, albeit with rejections $\sim 5 \%$ below those recorded for xylene due to the increased membrane swelling. It is noted that zero rejections were previously reported by van der Bruggen et $a l^{8}{ }^{8}$, who studied the behaviour of a $340 \mathrm{~g} \mathrm{~mol}^{-1}$ solute in a range of solvents with an MPF-50 membrane. They found that solute rejection was zero in n-hexane (but not in other solvents), and speculatively suggested that contact with organic solvents increases the mobility of the polymeric chains in the membrane to allow unhindered transport of solvent and solute.

The outlying rejection for rubrene in Figure 9 questions the validity of using molecular weight to characterise solute rejection. Due to the fine length scales involved in NF, the dimensions of the solute molecule are likely to be an important factor in determining rejection behaviour. Although there are inevitable approximations, representative sizes of the solute molecules were calculated using bond lengths and covalent radii and, where necessary, by resolving bond angles to a single plane. There are several dimensions that could be used to describe the size of a particular solute, for instance, the maximum length of the molecule or the diameter of a sphere that encloses the entire molecule. By way of example, the limiting rejection in xylene as a function of maximum length is shown in Figure 10; it is noted that the tested solvents have estimated maximum molecular dimensions of $0.8 \mathrm{~nm}$ or less. These data give a relatively sharp transition in the region of 1-2 $\mathrm{nm}$ and the result for rubrene follows the data for the other solutes closely. With experimentally measured rejections it is possible to use models developed for ultrafiltration to predict an equivalent pore size of the PDMS membrane ${ }^{11,12}$. Based on the rejection of 9,10 diphenylanthracene from xylene, the Ferry, Steric-Hindrance-Pore (SHP) and Verniory models respectively predict average pore sizes of $2.2 \mathrm{~nm}, 2.0 \mathrm{~nm}$ and $2.5 \mathrm{~nm}$, which are at the upper limit of the value suggested by Figure 10.

It is noted that the data in Figures 9 and 10 could be interpreted on the basis of solution-diffusion since the solute diffusion coefficient will decrease with increasing solute size. Although alternative mechanisms cannot be completely dismissed given the current level of knowledge, in the authors 
opinion the sharpness of the profile in Figure 10 is more indicative of a size exclusion/sieving mechanism. Considerable coincidence would be required for the sorption and diffusion coefficients of all the tested solutes to align in a manner to produce the results shown.

\section{Modelling}

In Figure 10, three distinct regions are evident. In Region $A$ the solvent induced swelling of the membrane causes sufficiently small solutes to translate directly with the solvent flow. In Region $C$ the solute molecules are sufficiently large for high rejection to occur predominantly via a size exclusion mechanism. In Region $B$ it is envisaged that a mixture of viscous flow and diffusion exists and a new model has been developed to aid interpretation over this region. ${ }^{*}$

In the viscous-diffusion model, solvent flux, $J_{s}$, is assumed viscous and expressed as

$J_{s}=\frac{k \Delta P}{x}$

where $\Delta P$ is the trans-membrane pressure, $x$ is membrane thickness and $k$ is the membrane permeability induced by the solvent (this parameter also incorporates the viscosity term). Solute flux, $J_{i}$, is considered a combination of viscous and diffusive transport and in the general case

$J_{i}=\frac{a C_{F} k \Delta P}{x}+\frac{(1-a) D\left(C_{F}-C_{P}\right)}{x}$

where $a$ is the fraction of solute undergoing transport with the viscous flow, $C_{F}$ and $C_{P}$ are solute concentrations in the feed and permeate respectively and $D$ is the diffusion coefficient. Solute rejection, $R=1-C_{P} / C_{F}$, but at low concentrations of solute, $C_{P}$ can be approximated as $J_{i} / J_{s}$, giving

$1-R=\frac{(1-a) D\left(C_{F}-C_{P}\right)+a C_{F} k \Delta P}{k C_{F} \Delta P}$

which upon dividing by $C_{F}$ and rearranging gives

$$
R=\frac{1-a}{1+\frac{(1-a) D}{k \Delta P}}
$$

In Equation (4), as $\Delta P \rightarrow \infty$ so $R \rightarrow(1-a)$ which is a different result from the solution-diffusion model ${ }^{5}$ where $R \rightarrow 1$ and is more in keeping with the behaviour of the solvent/solute systems in Region $B$ of Figure 10. Noting that the data were obtained above the hydrodynamic threshold, Figure 11 shows the experimental rejection of 9,10 diphenylanthracene along with the rejections predicted by the viscous-diffusion model. A least-squares analysis was used to fit the model to the experimental data and facilitate determination of the two parameters, $a$ and $D$; the former has most effect on the magnitude of the limiting rejection whilst $D$ principally influences the form (shape) of the rejection curve. Figure 11 shows the viscous-diffusion model to fit the experimental data well,

\footnotetext{
${ }^{* * *}$ The authors recognise that their data could potentially be analysed and interpreted using a range of models such as Spiegler-Kedem, Stefan-Maxwell, Solution-Diffusion etc. The use of the viscous-diffusion model is not intended to either degrade the applicability or validity of these models and given their growing wealth of experimental data, the authors intend to publish future papers on wider aspects of modelling.
} 
which in turn suggests that the concept of a combined viscous and diffusive solute flow may have some virtue; see also ${ }^{10,17}$. The value of $a$ is dependent on the solvent type (i.e. the degree of swelling) and the size of the solute molecule under consideration. The model suggests that larger solutes will be rejected by size-exclusion, or perhaps transport very slowly via a diffusive mechanism. In either case, solute rejection can be expected to be very high with correspondingly low values of diffusion coefficient. Similarly, small solutes will transport entirely with the viscous flow and undergo very little or no separation. The rejections observed in Figure 10 support these arguments. In the viscous flow Region $A$, the parameter $a=1$, and in the diffusive or size exclusion Region $C, a=0$ and $D$ values are very low. In the intermediate Region $B$, where the solute molecular weight is of the order of $250-400 \mathrm{~g} / \mathrm{mol}$, the viscous-diffusion model is able to predict the relationship between rejection and pressure with values of $a$ and $D$ varying accordingly.

\section{CONCLUSIONS}

The rejection of low-polarity organic solute compounds in organic solvents has been shown to be dependent on solute size, hydrodynamic conditions, operating pressure and the swelling effect of the solvent. A hydrodynamic threshold is considered to exist, above which solute rejection plateau's. For crossflow rates above the threshold, the swelling effect of the solvent was found to be the predominant factor governing solute rejection with a newly developed viscous-diffusion model providing a good correlation to the experimental data. The concept of viscous flow is supported by the observation that the rejection of low-polarity solutes is predominantly one of size exclusion. Poor swelling solvents yield a lower flux and higher solute rejection than good-swelling solvents. It is postulated that swelling increases the free volume within the membrane, which can be interpreted as an induced pore-like structure or raised porosity depending on the terminology used, and that the Hildebrand solubility parameter is a good indicator of swelling potential for PDMS membranes as well as a good predictor of their likely flux/rejection behaviour. The ability of the viscous-diffusion model to predict the solute rejection provides evidence that a supposedly dense PDMS membrane can exhibit the characteristics of a porous structure when swollen.

\section{ACKNOWLEDGEMENTS}

Shell Global Solutions (UK) are acknowledged for supplying the project funding, technical hardware and some materials. EPSRC are also acknowledged for part-funding this project. The PDMS membranes used in this study were kindly supplied by GKSS Forschungszentrum.

\section{NOMENCLATURE}

a Fraction of solute undergoing viscous flow

$C_{F} \quad$ Solute concentration in the feed (ppm)

$C_{P} \quad$ Solute concentration in the permeate $(\mathrm{ppm})$

$D \quad$ Diffusion coefficient $\left(\mathrm{m}^{2} / \mathrm{s}\right)$

$J_{i} \quad$ Solute flux $(\mathrm{m} / \mathrm{s})$

$J_{s} \quad$ Solvent flux $(\mathrm{m} / \mathrm{s})$

$k \quad$ Solvent induced permeability $\left(\mathrm{m}^{2} / \mathrm{Pa} \mathrm{s}\right)$

$\triangle P \quad$ Trans-membrane pressure $(\mathrm{Pa})$

$Q \quad$ Crossflow rate $(1 / \mathrm{min})$

$R \quad$ Solute rejection

$x \quad$ Membrane thickness $(\mathrm{m})$

$\delta \quad$ Solubility parameter $\left(\mathrm{MPa}^{0.5}\right)$

$\mu \quad$ Solvent viscosity ( $\mathrm{Pa} \mathrm{s})$

$\rho \quad$ Solvent density $\left(\mathrm{kg} / \mathrm{m}^{3}\right)$ 


\section{REFERENCES}

1. Scarpello J.T., Nair D., Freitas dos Santos L.M., White L.S. and Livingston A.G., 2002, The separation of homogeneous organometallic catalysts using solvent resistant nanofiltration, $J$. Membrane Science, 203, 71-85.

2. Zwijnenberg H.J., Krosse A.M., Ebert K., Peinemann K-V. and Cuperus F.P., 1999, Acetonestable nanofiltration membranes in deacidifying vegetable oil, J. American Oil Chemists Society, 76, 83-87.

3. Lonsdale H., Merten U. and Riley R., 1965, Transport of cellulose acetate osmotic membranes, J. Applied Polymer Science, 9, 1341-1362.

4. Favre E., Schaetzel P., Nguygen Q.T., Clement R. and Neel J., 1994, Sorption, diffusion and vapour permeation of various penetrants through dense poly (dimethylsiloxane) membranes: a transport analysis, J. Membrane Science, 92, 169-184.

5. Wijmans J.G. and Baker R.W., 1995, The solution-diffusion model: A review, J. Membrane Science, 107, 1-21.

6. Bhanushali D., Kloos S., Kurth C. and Bhattacharyya D., 2001, Performance of solventresistant membranes for non-aqueous systems: Solvent permeation results and modelling, $J$. Membrane Science, 189, 1-21.

7. Robinson J.P., Tarleton E.S., Millington C.R. and Nijmeijer A., 2004, Solvent flux through dense polymeric nanofiltration membranes, J. Membrane Science, 230, 29-37.

8. van der Bruggen B., Geens J. and Vandecasteele C., 2002, Fluxes and rejections for nanofiltration with solvent stable polymeric membranes in water, ethanol and n-hexane, Chemical Engineering Science, 57, 2511-2518.

9. Gibbins E., D'Antonio M., Nair D., White L.S., Freitas dos Santos L.M., Vankelecom I.F.J. and Livingston A.G., 2002, Observations on solvent flux and solute rejection across solvent resistant nanofiltration membranes, Desalination, 147, 307-313.

10. Bhanushali D., Kloos S. and Bhattacharyya D., 2002, Solute transport in solvent-resistant nanofiltration membranes for non-aqueous systems: Experimental results and the role of solute-solvent coupling, J. Membrane Science, 208(1-2), 343-359.

11. Raman L.P., Cheryan M. and Rajagopalan N, 1996, Solvent recovery and partial deacidification of vegetable oils by membrane technology, Fett-Lipid, 98(1), 10-14.

12. White L.S. 2002, Transport properties of a polyimide solvent resistant nanofiltration membrane, J. Membrane Science, 205(1-2), 191-202.

13. Stafie N., Stamatialis D.F. and Wessling M., 2003, Insight into the transport of hexane-solute systems through tailor-made composite membranes, J. Membrane Science, 228, 103-116.

14. Robinson J.P., Tarleton E.S., Millington C.R. and Nijmeijer A., 2004, Evidence for swellinginduced pore structure in dense PDMS nanofiltration membranes, FILTRATION, 4(1), 50-56.

15. van der Bruggen B., Schaep J., Wilms D. and Vandecasteele C., 2000, A comparison of models to describe the maximal retention of organic molecules in nanofiltration, Separation Science \& Technology, 35(2), 169-182. 
16. Nakao S-I. and Kimura S., 1981, Analysis of solute rejection in ultrafiltration, J. of Chemical Engineering Japan, 14(1), 32-37.

17. Paul D.R., Paciotti J.D. and Ebra-Lima O.M., 1975, Hydraulic permeation of liquids through swollen polymeric networks, J. Applied Polymer Science, 19, 1837-1845. 


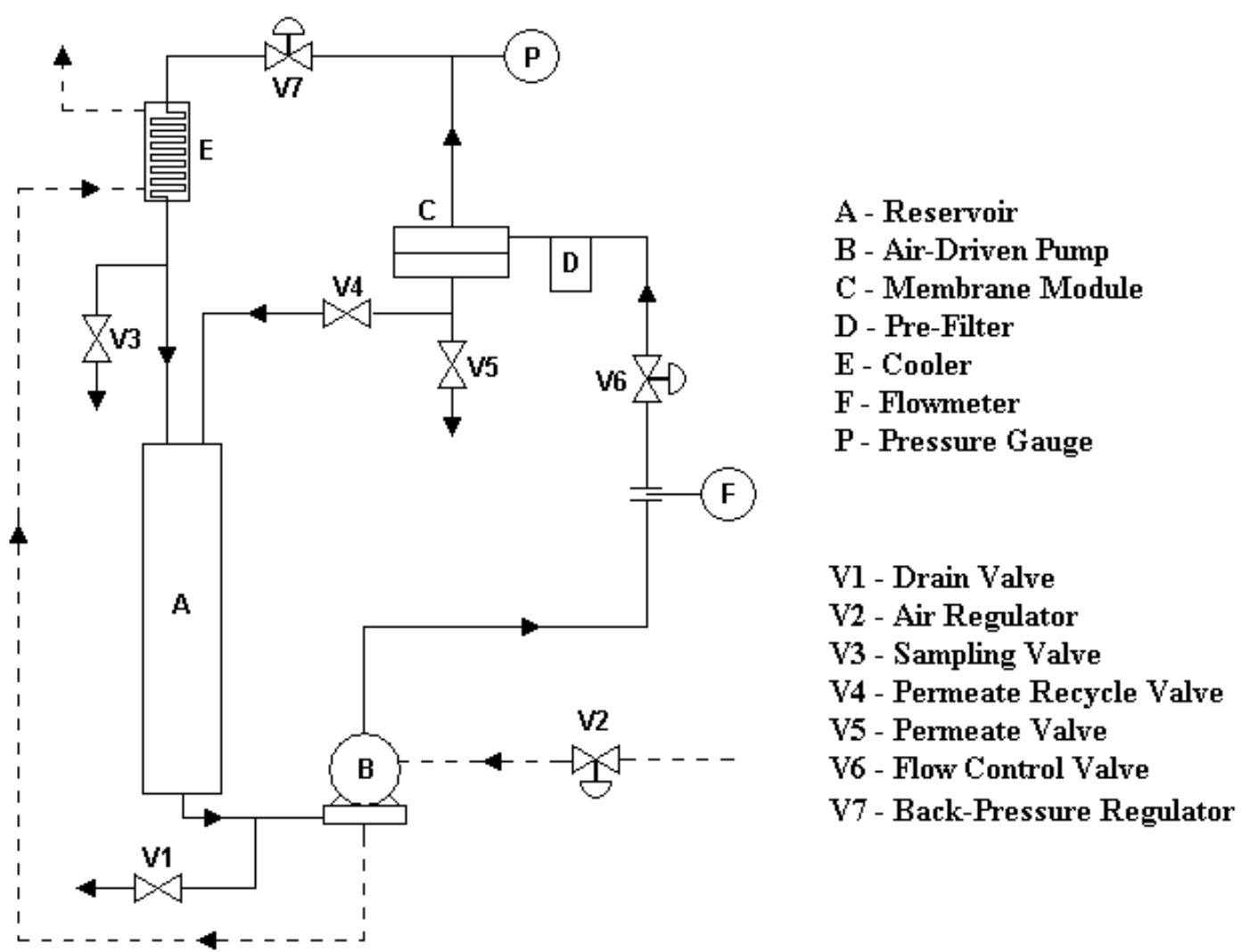

Figure 1: Schematic of the crossflow membrane filtration apparatus.<smiles>CC(=O)/C=C(/C)O[Ga](O/C(C)=C\C(C)=O)O/C(C)=C\C(C)=O</smiles><smiles>c1ccc(-c2c3ccccc3c(-c3ccccc3)c3ccccc23)cc1</smiles>

Figure 2: Representative structures of the principal solute compounds - iron (III) acetylacetonate (left) and 9,10 diphenylanthracene (right). 


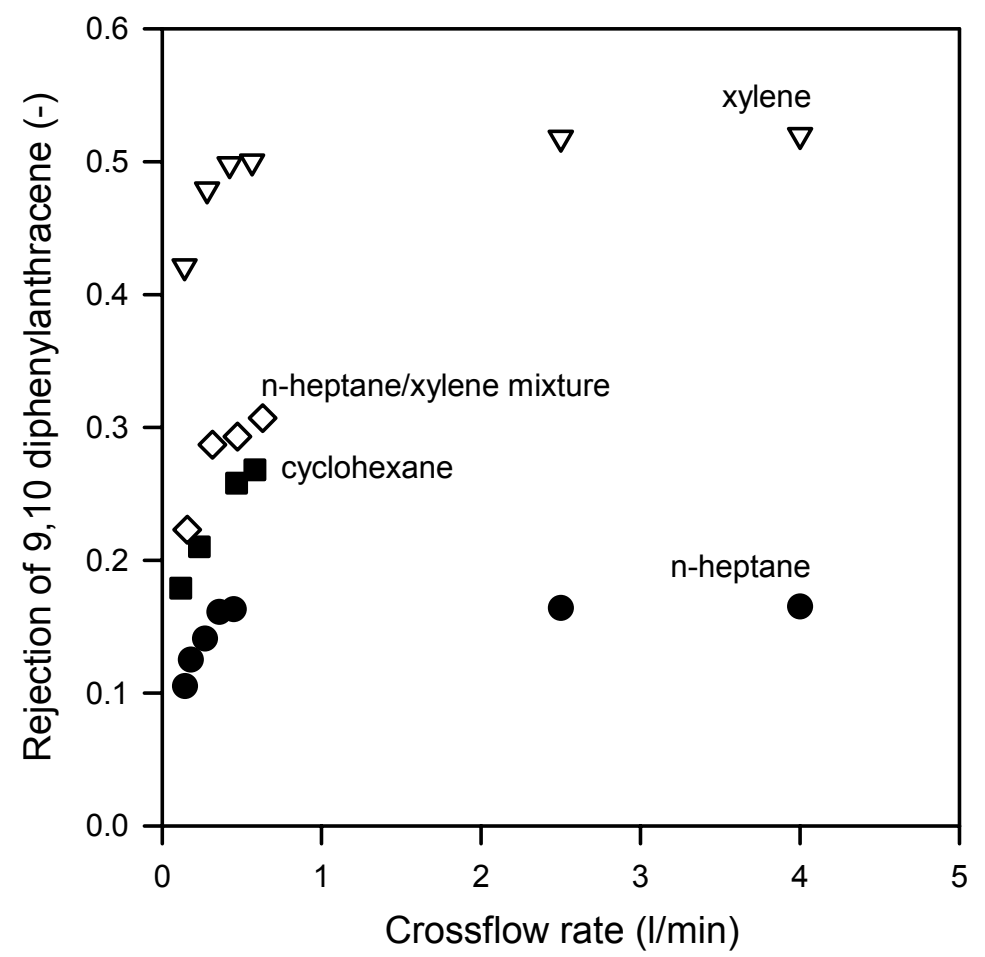

Figure 3: Effect of crossflow rate on the rejection of 9,10 diphenylanthracene from four solvents $\left(C_{F}\right.$ $=20 \mathrm{ppm}$ ).

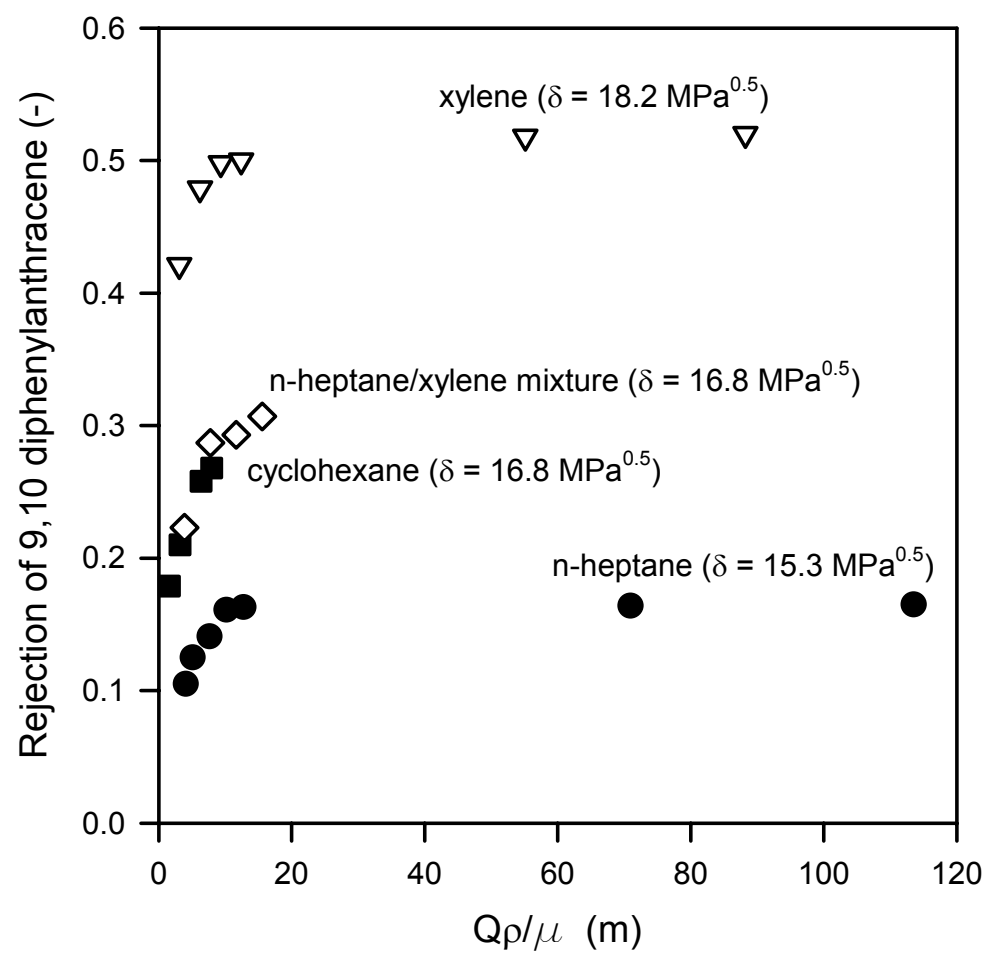

Figure 4: Effect of Reynolds Number (equivalent) on the rejection of 9,10 diphenylanthracene from four solvents $\left(C_{F}=20 \mathrm{ppm}\right)$. 


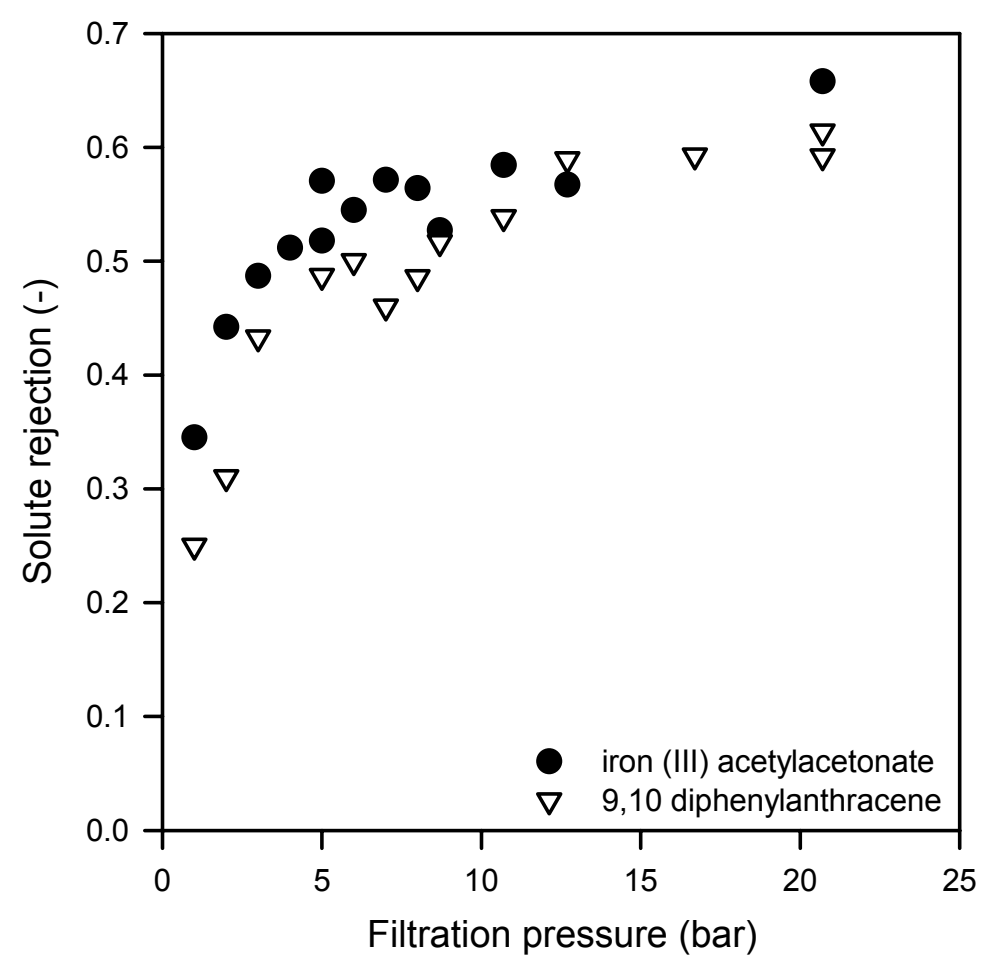

Figure 5: Rejections of iron (III) acetylacetonate and 9,10 diphenylanthracene from xylene over a range of pressures $\left(C_{F}=20 \mathrm{ppm}\right)$.

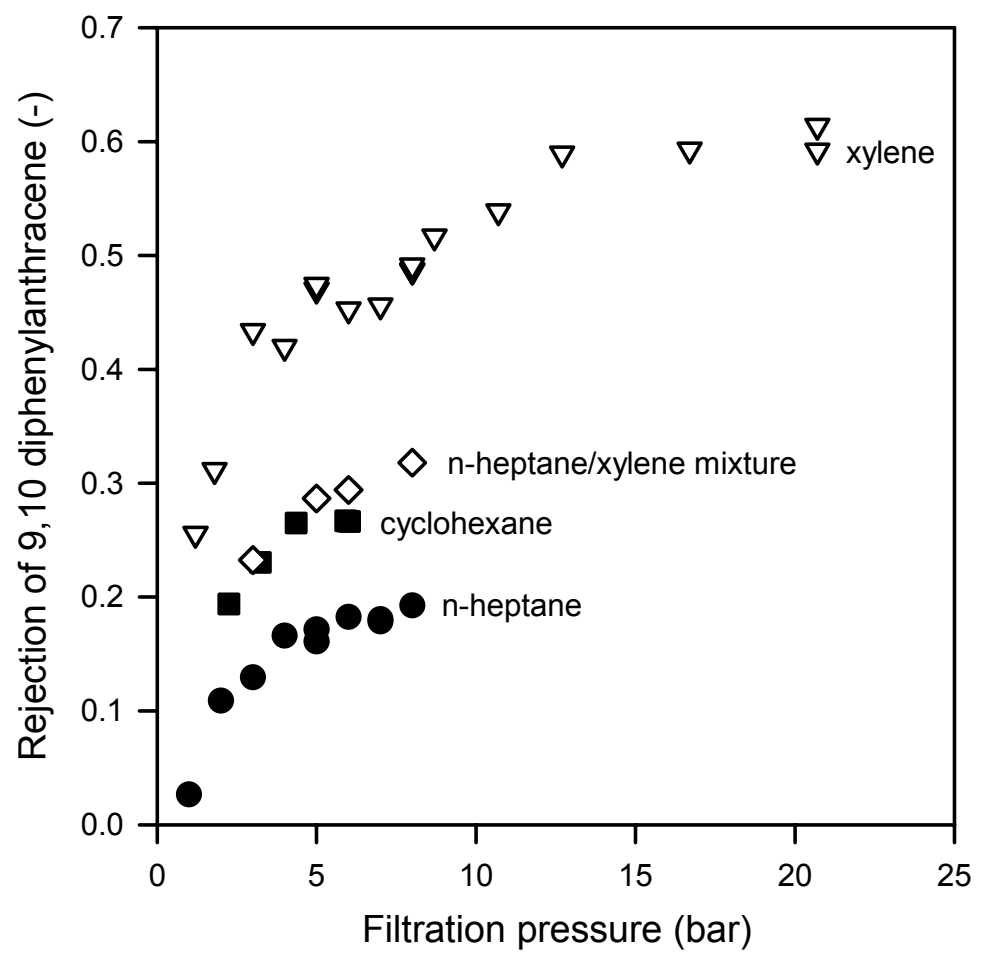

Figure 6: Effect of pressure on the rejection of 9,10 diphenylanthracene from four solvents $\left(C_{F}=20\right.$ ppm). 


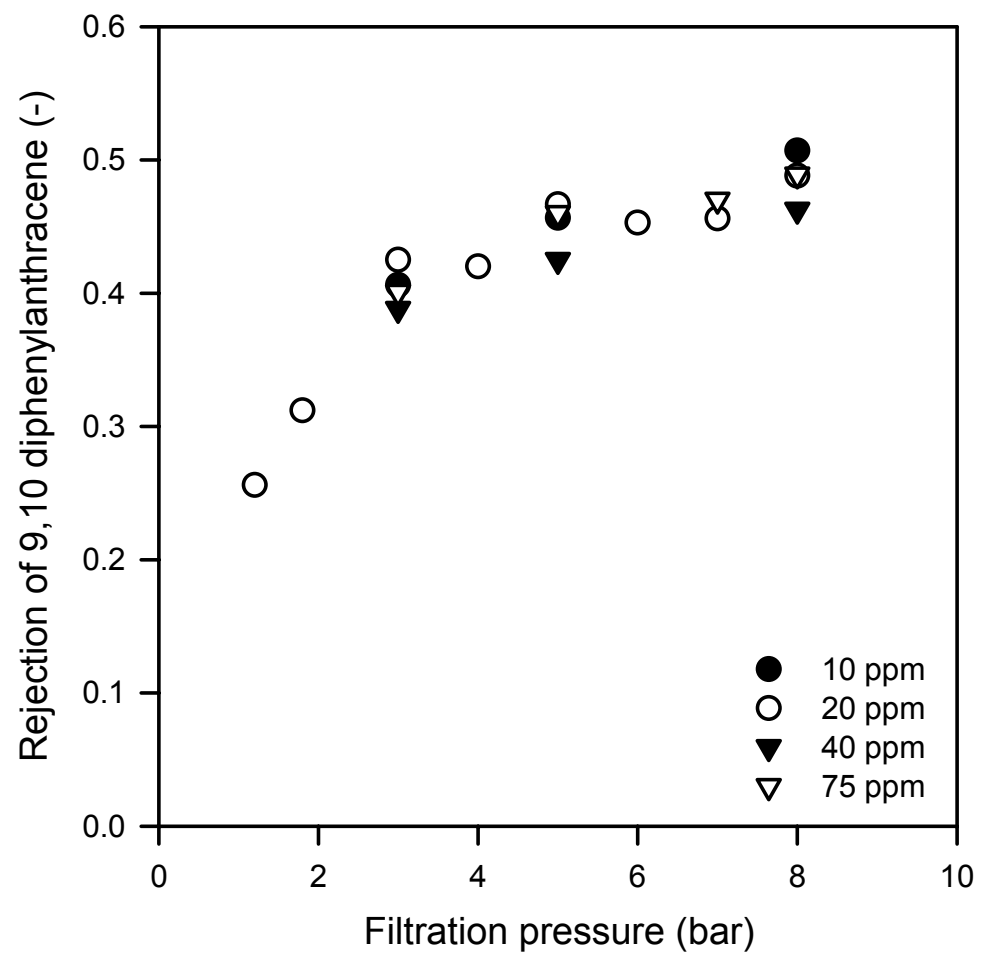

Figure 7: Typical effect of solute concentration on the rejection behaviour of 9,10 diphenylanthracene.

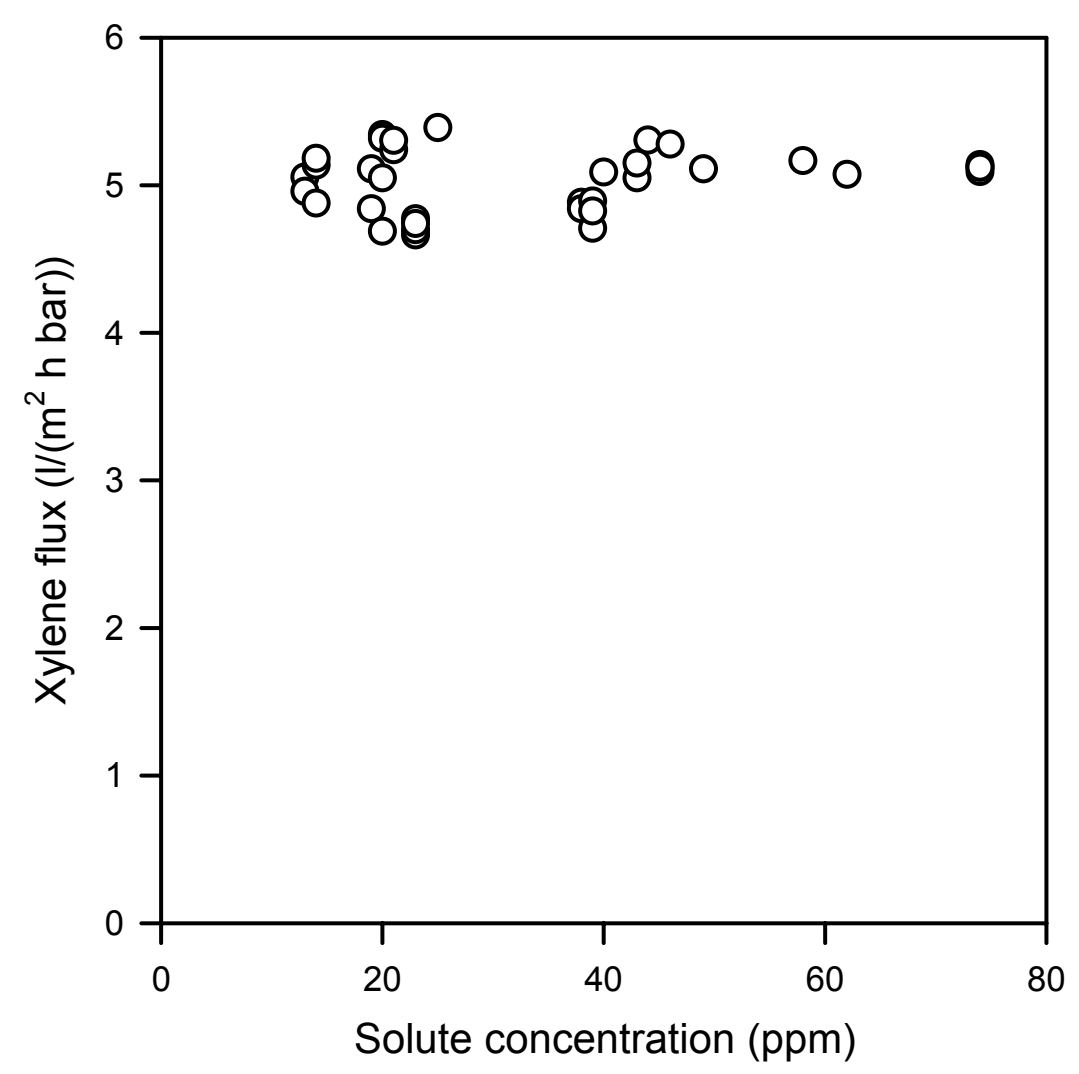

Figure 8: Influence of 9,10 diphenylanthracene solute concentration on xylene flux. 


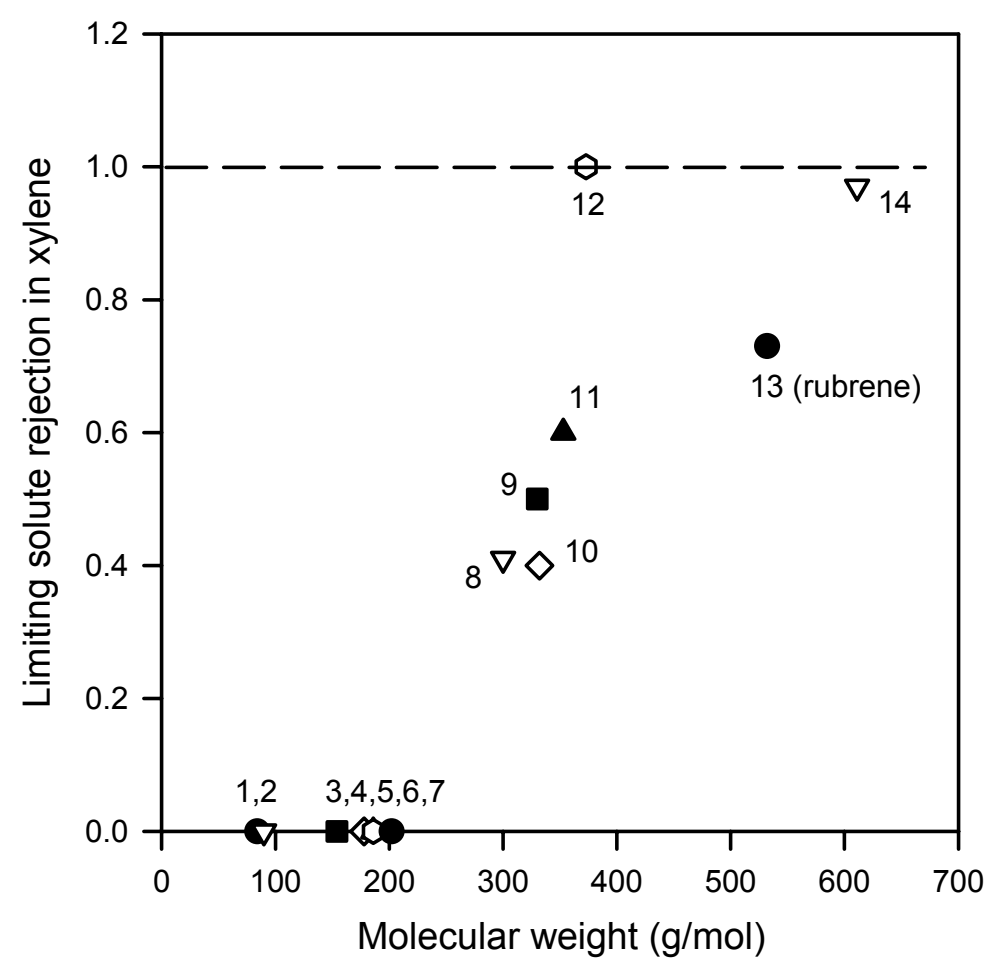

Figure 9: Effect of solute molecular weight on solute rejection from a xylene solvent $\left(C_{F}=25 \mathrm{ppm}\right.$; see Table 1 for solute designations).

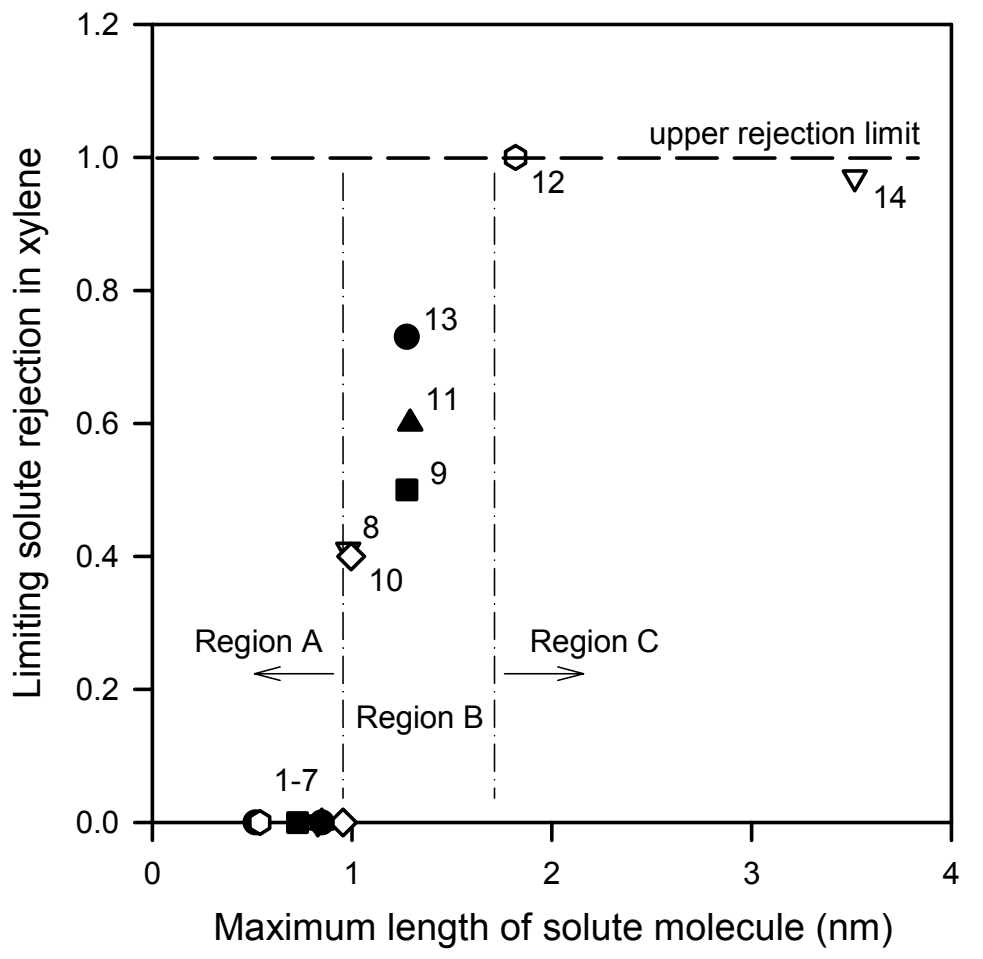

Figure 10: Effect of molecular dimension on solute rejection from a xylene solvent $\left(C_{F}=25 \mathrm{ppm}\right.$; see Table 1 for solute designations; Region A: viscous flow, no separation; Region $B$ : viscous and diffusive flow; Region $C$ : size exclusion, high rejection). 


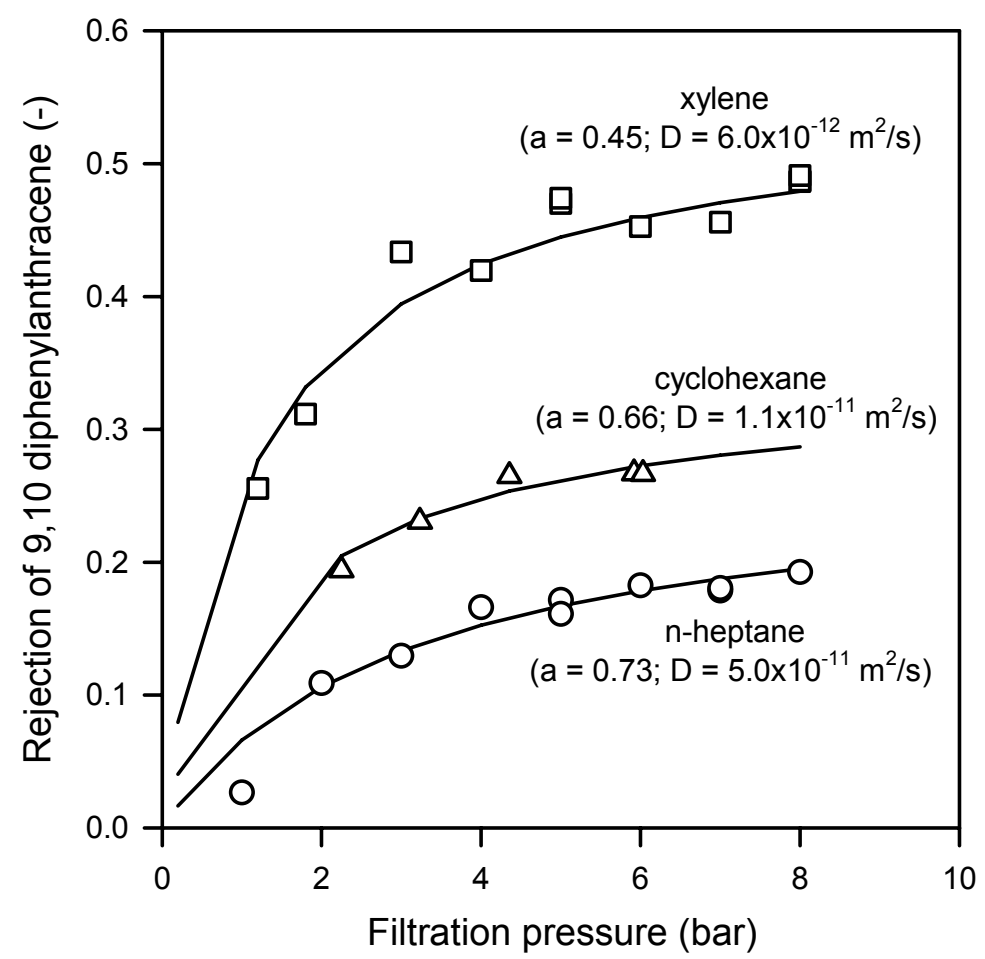

Figure 11: Rejection of 20 ppm 9,10 diphenylanthracene from three solvents and corresponding applications of the viscous-diffusion flow model.

\begin{tabular}{cccc}
\hline $\begin{array}{c}\text { Solute } \\
\text { designation }\end{array}$ & Solute name & Classification & $\begin{array}{c}\text { Molecular } \\
\text { weight }(\mathrm{g} / \mathrm{mol})\end{array}$ \\
\hline 1 & thiophene & thiophene & 84 \\
2 & 1-butanethiol & mercaptan & 90 \\
3 & acenaphthene & PNA & 154 \\
4 & anthracene & PNA & 178 \\
5 & phenanthrene & PNA & 176 \\
6 & ferrocene & OM & 186 \\
7 & pyrene & PNA & 202 \\
8 & coronene & PNA & 300 \\
9 & 9,10 diphenylanthracene & PNA & 330 \\
10 & $1,1,2,2$ tetraphenylethylene & PNA & 332 \\
11 & iron (III) acetylacetonate & OM & 353 \\
12 & iron (III) naphthenate & OM & 373 \\
13 & rubrene & PNA & 532 \\
14 & copper (II) naphthenate & OM & 612 \\
\hline
\end{tabular}

Table 1: Classification and molecular weight of test solutes (PNA $\equiv$ poly-nuclear aromatic; OM $\equiv$ organometallic). 